\title{
Compact Layer of Alkali Ions at the Surface of Colloidal Silica
}

\author{
Aleksey M. Tikhonov* \\ University of Chicago, Consortium of Advanced Radiation Sources, and Brookhaven National Laboratory, \\ National Synchrotron Light Source, Beamline X19C, Upton, New York 11973
}

Received: August 26, 2006; In Final Form: October 24, 2006

\begin{abstract}
The forces of electrical imaging strongly polarize the surface of colloidal silica. I used X-ray scattering to study the adsorbed 2-nm-thick compact layer of alkali ions at the surface of concentrated solutions of 5-nm, 7-nm, and 22-nm particles, stabilized by either $\mathrm{NaOH}$ or a mixture of $\mathrm{NaOH}$ and $\mathrm{CsOH}$, with the total bulk concentration of alkali ions ranging from 0.1 to $0.7 \mathrm{~mol} / \mathrm{L}$. The observed structure of the compact layer is almost independent of the size of the particles and the concentration of alkali base in the sol; it can be described by a two-layer model, that is, a $\sim 6-8$ - $\AA$-thick layer of directly adsorbed hydrated alkali ions with a surface concentration of $\sim 3 \times 10^{18} \mathrm{~m}^{-2}$, and a $\sim 13$ - $\AA$-thick layer with a surface concentration of sodium ions of $\sim 8$ $\times 10^{18} \mathrm{~m}^{-2}$. In cesium-enriched sols, $\mathrm{Cs}^{+}$ions preferentially adsorb in the first layer replacing $\mathrm{Na}^{+}$; their density in the second layer does not depend on the presence of cesium in the sol. The difference in the adsorption of $\mathrm{Cs}^{+}$and $\mathrm{Na}^{+}$ions can be explained by the ion-size-dependent term in the electrostatic Gibbs energy equation derived earlier by others. I also discuss the surface charge density and the value of surface tension at both the air/sol and the hexane/sol interfaces.
\end{abstract}

\section{Introduction}

Inorganic ions at the surface of aqueous electrolyte solutions play a key role in a wide variety of electrochemical processes at the liquid/vapor and liquid/liquid interfaces that have fundamental importance in biophysics, surface and colloidal chemistry, atmospheric chemistry, and energy storage systems. ${ }^{1-7}$ Frumkin $^{8,9}$ usually is credited with first pointing out that the surface tension and surface potential of aqueous solutions of inorganic electrolytes may depend on ionic radii. Indeed, for a long time, many authors discussed the properties of the air/ water surface in terms of the Wagner-Onsager-Samaras ${ }^{10,11}$ approximation, wherein ions are treated as point charges. Much later, Ulstrup and Kharkats ${ }^{12,13}$ showed that in the approximation of continuous media, ions with different radii interact in a different way with the boundary between two dielectric media. Recently, Markin and Volkov ${ }^{14}$ developed these ideas further in explaining quantitatively the macroscopic manifestations of the ion finite-size effects at the air/water interface.

Under certain conditions, surface X-ray reflectivity can provide information about the surface-normal structure of the liquid surface with spatial resolution down to $1 \AA .{ }^{15,16}$ The inplane structure of the liquid surface can be studied with similar spatial resolution by the grazing incidence diffraction technique. ${ }^{17-20}$ Alternatively, computational methods can reveal the equilibrium properties of the liquid surface and ion distributions across liquid-liquid interfaces with atomic resolution. ${ }^{21-23}$ Therefore, $\mathrm{X}$-ray scattering experiments provide a basis to validate theoretical models. For example, Luo et al. ${ }^{24}$ showed that the ion distributions of organic ions, established from an X-ray reflectivity experiment at the liquid-liquid interface, can be explained by a generalized Poisson-Boltzmann equation without adjustable parameters.

In this work, I studied ion finite-size effect in the compact layers of alkali ions adsorbed at the surface of concentrated

\footnotetext{
*E-mail: tikhonov@bnl.gov.
}

aqueous solutions of colloidal silica, stabilized by a small amount of an alkali base. ${ }^{25}$ In the exceptionally wide electrical double layer at the surface of silica sol, the surface compact layer is well separated from the anionic colloidal particles, which significantly facilitates interpreting the X-ray scattering data. ${ }^{26,27}$ The pronounced wideness of the transition region at the surface of colloidal silica reflects the extremely large difference between the forces of electrical imaging for nanoparticles and alkali ions. This difference can be understood qualitatively in terms of the "classical" energy of the "image force" for the point charge, $Z$, placed into the dielectric media with permittivity, $\epsilon_{1}$ at the distance, $h$, from the planar boundary with another dielectric media with permittivity $\epsilon_{2}{ }^{10-11,28}$

$$
\frac{1}{4 \pi \epsilon_{0}} \frac{Z^{2}}{4 \epsilon_{1}} \frac{\epsilon_{1}-\epsilon_{2}}{\epsilon_{1}+\epsilon_{2}} \frac{1}{h}
$$

where $\epsilon_{0}=8.85 \times 10^{-12} \mathrm{~F} / \mathrm{m}$ is the dielectric permittivity of the vacuum.

For a concentrated solution of particles of $\sim 10 \mathrm{~nm}$ diameter $(D)$, the equilibrium charge density at the silica surface at $\mathrm{pH}$ $=10$ is as large as $\sim 0.5 \mathrm{C} / \mathrm{m}^{2}$ because of deprotonation of the silanol groups by the hydroxyl ions, which is associated with a significant energy gain of $\sim 7 k_{\mathrm{B}} T$ per ion ( $k_{\mathrm{B}}$ is Boltzmann's constant). Because the dielectric permittivities of water and air are $\epsilon_{1}=78$ and $\epsilon_{2}=1$, respectively, then $\epsilon_{1}-\epsilon_{2}>0$ so that both alkali ions and nanoparticles in the solution are repelled from the air/sol interface by their electrical images. A particle in the sol can be considered, to certain extent, as a super ion (anion) because it is carrying a very large adsorbed charge, $Z$ $\approx 10^{3} e$ ( $e$ is the elementary charge), and its energy term (eq 1$)$ is 6 orders of magnitude larger than that for an alkali ion. Therefore, separation of electrical charges at the interface is unavoidable. However, eq 1 by itself does not explain the equilibrium structure of the interface because it does not account for the polarization of the interface, the changes in the dielectric 


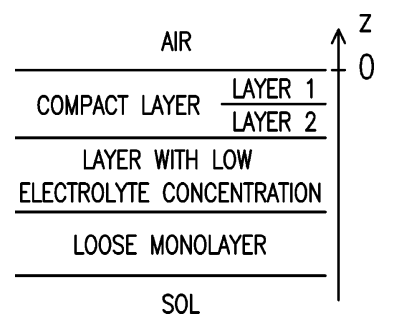

Figure 1. Four-layer model for the transition region at the air/sol interface.

properties of the media in the transition region, the particleparticle and ion-particle interactions, and the like.

Earlier, Madsen et al. ${ }^{29}$ using X-ray scattering to explore the surface of colloidal solutions of silica particles of $D>30 \mathrm{~nm}$, and unspecified alkalinity, showed that the colloidal particles do not congregate directly at the sol's surface. However, the layering model they proposed, based on the X-ray reflectivity data with spatial resolution $2 \pi / q_{z}^{\max } \approx 100 \AA$, does not describe the adsorption of the compact layer at the sol's surface. My recent findings from X-ray scattering experiments $(2 \pi /$ $q_{z}^{\max } \approx 20 \AA$ ) at interfaces between $n$-hexane and nanocolloidal solutions of silica stabilized by $\mathrm{NaOH}(\mathrm{pH} \approx 9-10)$ revealed a surface structure that I described by a three-layer model, that is, a compact layer of $\mathrm{Na}^{+}$at the hexane/sol interface, a loose monolayer of nanocolloidal particles as part of a thick diffuse layer, in between which was a layer with low electrolyte concentration (see Figure 1). ${ }^{26,27}$ The plane of the closest approach of the nanoparticles is situated at $\sim 10-20 \mathrm{~nm}$ from the hexane's interface, so that the 2-4-nm-thick compact layer is separated from the countercharge by $\sim 6-10$-nm-thick layer of "surface water". Depending on the model for the surfacenormal structure, the estimated surface density, $\Gamma^{+}$, of $\mathrm{Na}^{+}$in the compact layer at the hexane/sol interface ranged between $\sim 2 \times 10^{18} \mathrm{~m}^{-2}$ and $\sim 6 \times 10^{18} \mathrm{~m}^{-2}$. According to a semiquantitative interface model reviewed by Vorotyntsev et al., ${ }^{30}$ the surface of the solution and the plane of the closest approach for nanoparticles can be considered as two individual interfaces, contributing independently to the potential drop across the interface.

At $\mathrm{pH}>11, \mathrm{Na}^{+}$cations cause amorphous silica hydrosols to coagulate, whereas $\mathrm{Cs}^{+}$cations are ineffective. ${ }^{31}$ Therefore, silica solutions can be further stabilized with very high bulk concentrations of $\mathrm{Cs}^{+}(\mathrm{pH}>11)$ by dissolving $\mathrm{CsOH}$ in an $\mathrm{NaOH}$-stabilized sol ( $\mathrm{pH} \approx 10$ ). Then, the contrast of the compact layer increases so much that precise information about its structure can be obtained by directly studying the sol's surface. In addition, the in-plane structure of the air/sol interface can be explored by a grazing incidence diffraction technique that is not applicable at the hexane/hydrosol surface because of the very strong scattering in the oil's bulk.

In this paper I describe my studies, via X-ray scattering, of the adsorbed compact layers of alkali ions at the surface of the concentrated solutions of 5-nm, 7-nm, and 22-nm particles stabilized by either $\mathrm{NaOH}$ or a mixture of $\mathrm{NaOH}$ and $\mathrm{CsOH}$. The former are very similar to the sols that were studied earlier in ref 27. The sizes of silica particles were chosen such that the information about the entire surface-normal structure could be obtained from a single X-ray reflectivity experiment. I report $\mathrm{X}$-ray data with a spatial resolution, $2 \pi / q_{z}^{\max }<10 \AA$, that can be described by a four-layer model, wherein the compact layer consists of two layers, that is, a layer 1 of directly adsorbed hydrated ions and a layer 2 of space charge (see Figure 1). The difference between my previous model and the present one of
TABLE 1: Parameters of Cesium-Doped Solutions of Colloidal Silica ${ }^{a}$

\begin{tabular}{llllllll}
\hline $\begin{array}{c}D \\
(\AA)\end{array}$ & $c_{\mathrm{b}}\left(\mathrm{m}^{-3}\right)$ & $\mathrm{pH}$ & $\begin{array}{c}f_{\mathrm{w}} \\
(\%)\end{array}$ & $\begin{array}{c}\xi \\
\left(\mathrm{g} / \mathrm{cm}^{3}\right)\end{array}$ & $\rho_{\mathrm{b}} / \rho_{0}$ & $\begin{array}{c}c_{\mathrm{Na}}^{+} \\
(\mathrm{mol} / \mathrm{L})\end{array}$ & $\begin{array}{c}c_{\mathrm{Cs}}^{+} \\
(\mathrm{mol} / \mathrm{L})\end{array}$ \\
\hline 50 & $\sim 2 \times 10^{23}$ & 12.2 & 85 & 1.12 & $1.17 \pm 0.01$ & $\sim 0.1$ & $\sim 0.5$ \\
70 & $\sim 2 \times 10^{23}$ & 11.6 & 70 & 1.20 & $1.18 \pm 0.01$ & $\sim 0.2$ & $\sim 0.2$ \\
220 & $\sim 6 \times 10^{22}$ & 11 & 60 & $\sim 1.3$ & $1.22 \pm 0.01$ & $\sim 0.05$ & $\sim 0.1$
\end{tabular}

${ }^{a} D$ is the diameter of the particles; $c_{\mathrm{b}}$ is the bulk concentration of the nanoparticles; $f_{\mathrm{w}}$ is the water content in the sol; $\xi$ is the specific gravity of the sol; $\rho_{\mathrm{b}} / \rho_{0}$ is normalized to the density of bulk water at normal conditions to the bulk electron density of the sol $\left(\rho_{0}=0.333\right.$ $\left.\mathrm{e}^{-} / \AA^{3}\right)$; and $c_{\mathrm{Na}}^{+}$and $c_{\mathrm{Cs}}^{+}$are the bulk concentrations of sodium and cesium in the sol.

the structure of the compact layer at the sol's surface and at the hexane/sol interface for $\mathrm{NaOH}$-stabilized sols lies in the better spatial resolution in the latter of the data collected at the air/sol interface, the much higher electron density of the compact layer in cesium-enriched sols, and the much smaller capillary-waveinduced surface roughness, $\sigma_{\text {cap }}$, at the surface of the sol than at the hexane/sol interface. The four-layer model is in accordance with the effect discussed by Ulstrup and Kharkats that explains segregation of the ions with different radii into two layers at the sol's surface. ${ }^{12-13}$

\section{Experimental Section}

Here, I report my findings on the surface-normal structure of sols with $9 \leq \mathrm{pH} \leq 12.5$ where the bulk concentration of cesium, $c_{\mathrm{Cs}}^{+} \approx 0.05-0.5 \mathrm{~mol} / \mathrm{L}$, is roughly the same as, or larger than the sodium concentration. I prepared cesium-enriched sols by mixing, in an ultrasonic bath (Branson 2510), a 1:1 (by weight) solution of cesium hydroxide $(99.95 \%$ on metal basis, Sigma-Aldrich) in deionized water (Barnstead UV) with $\mathrm{NaOH}-$ stabilized suspensions of colloidal silica. The concentrated homogenized sols, stabilized by sodium hydroxide (supplied by Grace Davison), contained silica particles with diameters of approximately $50 \AA$ (Ludox FM, pH $\approx 10$ ), $70 \AA$ (Ludox SM$30, \mathrm{pH} \approx 10$ ), and $220 \AA$ (Ludox TM-40, $\mathrm{pH} \approx 9) .32$ These solutions had specific gravities, $\xi$, respectively, of $1.068 \pm 0.004$ $\mathrm{g} / \mathrm{cm}^{3}$ (16\% of $\mathrm{SiO}_{2}$ and $0.3 \%$ of $\mathrm{Na}$ by weight), $1.177 \pm 0.004$ $\mathrm{g} / \mathrm{cm}^{3}$ (30\% of $\mathrm{SiO}_{2}$ and $0.5 \%$ of $\mathrm{Na}$ by weight), and $1.257 \pm$ $0.004 \mathrm{~g} / \mathrm{cm}^{3}$ (40\% of $\mathrm{SiO}_{2}$ and $0.3 \%$ of $\mathrm{Na}$ by weight). Their specific surface areas were $\sim 5 \times 10^{5} \mathrm{~m}^{2} / \mathrm{kg}$ (Ludox FM), $\sim 3$ $\times 10^{5} \mathrm{~m}^{2} / \mathrm{kg}$ (Ludox SM-30), and $\sim 10^{5} \mathrm{~m}^{2} / \mathrm{kg}$ (Ludox TM$40)$.

The molar concentration of free hydroxyl ions in the bulk of these sols is very small, $c^{-} \approx 10^{-4}-10^{-5} \mathrm{~mol} / \mathrm{L}$, compared with the bulk concentration of sodium ions $c_{\mathrm{Na}}^{+}=f_{\mathrm{Na}} \xi / M_{\mathrm{Na}} \approx$ $0.1-0.2 \mathrm{~mol} / \mathrm{L}\left(M_{\mathrm{Na}} \approx 23 \mathrm{~g} / \mathrm{mol}\right.$ is the atomic weight of $\mathrm{Na}$, and $f_{\mathrm{Na}}$ is the weight fraction of sodium in the suspension) because the $\mathrm{OH}^{-}$ions are adsorbed at the surface of the silica particles. At high concentrations of alkali base $(\mathrm{pH}>12)$, the Ludox sols usually appear opaque, and transform into silica gel due to the coalescence of the nanoparticles. Table 1 lists parameters of the cesium-enriched sols with $c_{\mathrm{Cs}}^{+} \approx 0.1-0.5$ $\mathrm{mol} / \mathrm{L}(\mathrm{pH}>11)$.

I carried out the X-ray scattering experiments at beamline X19C, National Synchrotron Light Source, Brookhaven National Laboratory ${ }^{33}$ employing a monochromatic focused X-ray beam $(\lambda=0.825 \pm 0.002 \AA)$ to explore the solutions' planar surface. Liquid samples were studied in a $\sim 50 \mathrm{~mL}$ capacity glass or polyethylene dish with a circular interfacial area $(100 \mathrm{~mm}$ diameter) that was placed inside a single-stage thermostat and mounted above the level of water in a bath $(\sim 200 \mathrm{~mm}$ diameter $)$, which served as a humidifier in the thermostat. Because cesium 


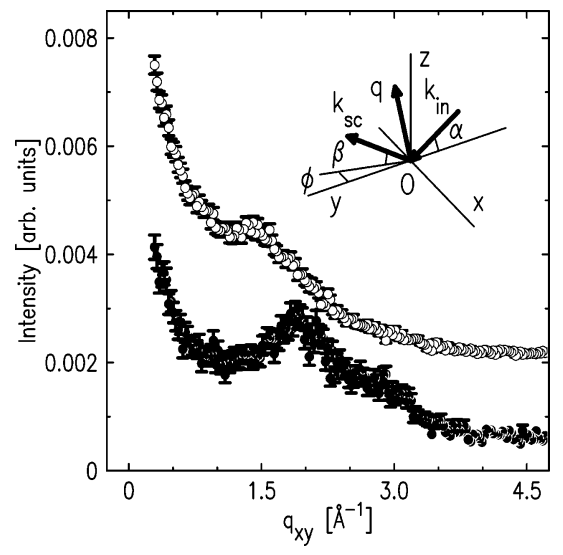

Figure 2. Scattering background at ambient conditions from the surface of $\mathrm{NaOH}$-stabilized sol with 7-nm particles (circles) and the surface of deionized and degassed water (dots). The recordings were taken at a glancing angle $\alpha \approx 0.07^{\circ}$ with a vertical position-sensitive detector (Ordela) by summing 30 channels covering the range of $\beta$ from 0.05 to $0.2^{\circ}\left(\Delta q_{z}=0.05 \AA^{-1}\right)$. The horizontal resolution of the detector in this experiment, which was set by Soller slits, was as much as $\Delta \phi=$ $0.2^{\circ}$. The inset is a sketch of the kinematics of the scattering at the silica sol's surface. The $x-y$ plane coincides with the interface, the $x$ axis is perpendicular to the beam's direction, and the $z$ axis is directed normal to the surface opposite to the gravitational force. $\mathbf{k}_{\mathrm{in}}$ and $\mathbf{k}_{\mathrm{sc}}$ are, respectively, wave vectors of the incident beam and beam scattered toward the point of observation, and $\mathbf{q}$ is the wave-vector transfer, $\mathbf{q}$ $=\mathbf{k}_{\text {in }}-\mathbf{k}_{\mathrm{sc}}$.

hydroxide slowly corrodes glass, all cesium-enriched samples were contained in high-density polyethylene dishes. Although exposed to air, colloidal silica adsorbs $\mathrm{CO}_{2}$ and the surfacenormal structure of the sol long remains stable; in fact, the X-ray reflectivity curves were reproducible within error bars for $\sim 48$ $\mathrm{h}$, during which time the bulk concentration of hydroxyl ions in the sample liquid fell 2-fold $(\Delta \mathrm{pH}<0.3) .{ }^{34}$ All X-ray scattering measurements were carried out after the samples were equilibrated at $T=298 \mathrm{~K}$ for at least $2 \mathrm{~h}$.

It is useful to describe the kinematics of scattering in the right-handed rectangular coordinate system where the origin, $O$, is in the center of the X-ray footprint; here, the $x y$ plane coincides with the air/sol interface, the axis $x$ is perpendicular to the beam's direction, and the axis $z$ is directed normal to the interface opposite to the gravitational force (see the inset in Figure 2). At the reflectivity condition, $\alpha=\beta$, and $\phi=0, \alpha$ is the incident angle in the $y z$ plane, $\beta$ is the angle in the vertical plane between the scattering direction and the interface, and $\phi$ is the angle in the $x y$ plane between the incident beam's direction and the direction of scattering. Because the angles $\alpha$ and $\beta$ were small in the experiment, at small-angle deviations, $\delta \phi$ and $\delta \beta$, from the specular conditions, the components of wave-vector transfer, $q$, can be written in the following form:

$$
\begin{gathered}
q_{x} \approx \frac{2 \pi}{\lambda} \delta \phi \\
q_{y} \approx \frac{2 \pi}{\lambda} \alpha \delta \beta \\
q_{z} \approx \frac{2 \pi}{\lambda}(\alpha+\beta)
\end{gathered}
$$

At the reflectivity conditions, there is only one component of the wave-vector transfer, $q_{z} \approx(4 \pi / \lambda) \alpha$. The scattering intensity under grazing incidence conditions is usually expressed as a function of $q_{x y}=\left(q_{x}^{2}+q_{y}^{2}\right)^{1 / 2}$. If $\alpha, \beta \ll 1$, then $q_{x y}=(4 \pi / \lambda)$ $\sin (\phi / 2)+o(\alpha)+o(\beta)$.

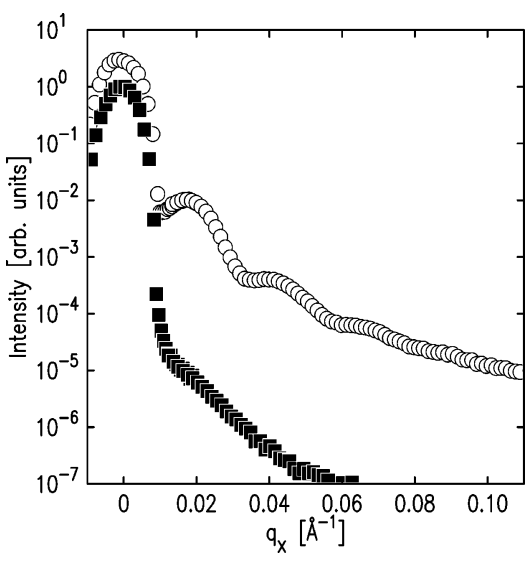

Figure 3. Bulk small-angle scattering below and above critical angle from the silica sol with 22-nm particles: squares $\alpha=0.8 \alpha_{\mathrm{c}} \approx 0.07^{\circ}$; circles $\alpha \sim \alpha_{c} \approx 0.09^{\circ}$. The central peak at $q_{x}=0$ is the reflected beam. At incident angles $\alpha \sim \alpha_{c}$, the penetration depth increases so much that the strong bulk small-angle scattering background consists of several concentric rings around the direction of the transmission beam. The radius of principal ring of small-angle scattering in $q$-space, $q_{\mathrm{o}}$, can be used to estimate the particle-particle distance in the bulk of the solution, $d_{\mathrm{b}} \approx 1.23\left(2 \pi / q_{\mathrm{o}}\right) \approx 250 \AA$. Because $q_{x \mathrm{o}} \approx 0.02 \AA^{-1}$ and $q_{\mathrm{c}} \approx 0.024 \AA^{-1}$ at $\alpha=\beta \sim \alpha_{\mathrm{c}}$, then $q_{\mathrm{o}} \approx \sqrt{q_{x \mathrm{o}}^{2}+q_{\mathrm{c}}^{2}} \approx 0.03 \AA^{-1}\left(q_{x y} \approx\right.$ $\left.q_{x}\right)$.

At incident angles below the critical angle, $\alpha_{c}$, the penetration of the X-rays is very shallow and scattering occurs in the top $\lambda /\left(2 \pi \alpha_{c}\right) \sim 80 \AA$ thick layer $\left(\alpha<0.8 \alpha_{c}\right)$. ${ }^{19,35} \alpha_{c}=$ $\lambda \sqrt{r_{\mathrm{e}} \rho_{\mathrm{b}} / \pi} \approx 0.09^{\circ}$, where $r_{\mathrm{e}}=2.814 \times 10^{-5} \AA$ is the electron's Thompson scattering length. The bulk electron densities, $\rho_{\mathrm{b}}$, of the $\mathrm{NaOH}$-stabilized sols were established with high accuracy from the density and weight of the silica per unit volume of the sol dried and annealed at high temperature. Normalized to the density of bulk water at normal conditions, $\rho_{0}=0.333 \mathrm{e}^{-} / \AA{ }^{3},{ }_{\mathrm{b}} / \rho_{0}=1.06 \pm 0.01$ for the solution of $50-\AA$ particles, with $1.15 \pm 0.01$, and $1.21 \pm 0.01$ for sols with $70-\AA$ and $220-\AA$ particles, correspondingly. Then, the $\rho_{\mathrm{b}}$ of the cesium-enriched sols was estimated from their density and known chemical composition (Table 1).

The surface of all NaOH-stabilized sols at ambient conditions scatters a grazing incident beam in a similar way: the scattering background contains a broad peak at $q_{x y}^{\prime} \approx 1.5 \AA^{-1}$, which is $\sim 0.5 \AA^{-1}$ wide. This peak was observed in the range of $q_{z}$ from 0 to $0.5 \AA^{-1}$. The circles in Figure 2 depict the diffraction data from the surface of the hydrosol with 7 -nm particles. The intensity of scattering was recorded at grazing angle $\alpha \approx 0.07^{\circ}$ with a vertical position-sensitive detector (Ordela) by summing 30 channels covering the range of $\beta$ from 0.05 to $0.2^{\circ}\left(\Delta q_{z}=\right.$ $0.05 \AA^{-1}$ ). The horizontal resolution of the detector in this experiment was as much as $\Delta \phi=0.2^{\circ}$. For comparison, I also measured the intensity of scattering from the surface of deionized and degassed water (the dots in Figure 2). Similar to the bulk background, it has a strong diffraction peak at $\sim 2 \AA^{-1}$, which is associated mainly with the $\mathrm{O}-\mathrm{O}$ correlations in water. ${ }^{36}$

At incident angles $\alpha \geq \alpha_{c}$, the penetration of the beam increases to considerable depths so that both the sol's surface and its bulk contribute to scattering. Bulk scattering is shown by the several concentric rings in the $q_{x} q_{y}$ plane around the direction of the transmission beam (Figure 3). ${ }^{26}$ The radius of the principal ring of small-angle scattering in the $q_{x} q_{y}$ plane, $q_{\mathrm{o}}$, can be used to estimate the particle-particle distance in the bulk of the solution, $d_{\mathrm{b}} \approx 1.23\left(2 \pi / q_{\mathrm{o}}\right) \cdot{ }^{37}$ Thus, the bulk concentration of nanoparticles in the sols, $c_{\mathrm{b}} \approx 1 / d_{\mathrm{b}}^{3}$, can be assessed from the position of the principal ring of the bulk small- 
TABLE 2: Estimates of the Parameters for the Four-Layer Model (See Figure 1) ${ }^{a}$

\begin{tabular}{rcccccccccc}
\hline$D(\AA)$ & $l_{1}(\AA)$ & $l_{2}(\AA)$ & $l_{3}(\AA)$ & $l_{4}(\AA)$ & $\rho_{1} / \rho_{0}$ & $\rho_{2} / \rho_{0}$ & $\rho_{3} / \rho_{0}$ & $\rho_{4} / \rho_{0}$ & $\sigma_{3}(\AA)$ & $\sigma_{3}(\AA)$ \\
\hline 50 & $7 \pm 2$ & $12 \pm 3$ & $100 \pm 10$ & $80 \pm 20$ & $0.35 \pm 0.07$ & $1.28 \pm 0.06$ & $1.08 \pm 0.01$ & $1.15 \pm 0.02$ & $19 \pm 3$ & $19 \pm 3$ \\
70 & $6.0 \pm 0.8$ & $12 \pm 3$ & $60 \pm 20$ & $75 \pm 5$ & $0.42 \pm 0.08$ & $1.24 \pm 0.05$ & $1.10 \pm 0.02$ & $1.33 \pm 0.04$ & $19 \pm 3$ & $18 \pm 3$ \\
220 & $5.6 \pm 0.5$ & $14 \pm 3$ & $140 \pm 20$ & $160 \pm 30$ & $0.7 \pm 0.2$ & $1.25 \pm 0.02$ & $1.15 \pm 0.02$ & $1.31 \pm 0.02$ & $40 \pm 10$ & $25 \pm 8$ \\
70 & & \multicolumn{7}{c}{ cesium-enriched sols } \\
50 & $8.3 \pm 0.3$ & $12.2 \pm 0.8$ & $40 \pm 20$ & $180 \pm 50$ & $0.9 \pm 0.04$ & $1.32 \pm 0.04$ & $1.14 \pm 0.05$ & $1.25 \pm 0.03$ & $20 \pm 10$ & $70 \pm 30$ \\
70 & $8.1 \pm 0.3$ & $13.6 \pm 0.5$ & $60 \pm 20$ & $60 \pm 10$ & $0.85 \pm 0.03$ & $1.26 \pm 0.01$ & $1.08 \pm 0.02$ & $1.30 \pm 0.05$ & $20 \pm 5$ & $25 \pm 4$ \\
220 & $8.0 \pm 0.3$ & $13 \pm 1$ & $140 \pm 20$ & $160 \pm 40$ & $0.84 \pm 0.03$ & $1.29 \pm 0.02$ & $1.13 \pm 0.02$ & $1.36 \pm 0.04$ & $50 \pm 10$ & $30 \pm 10$
\end{tabular}

${ }^{a} l_{i}$ are the thicknesses of the interfacial layers with electron densities $\rho_{i} / \rho_{0}$, normalized to the density of bulk water at normal conditions $\left(\rho_{0}=\right.$ $\left.0.333 \mathrm{e}^{-} / \AA^{3}\right)$. The interfacial widths $\sigma_{0}=\sigma_{1}=\sigma_{2}=\sigma_{\text {cap }}\left(\sigma_{\text {cap }}=2.6 \pm 0.2 \AA\right) . \sigma_{3}$ is the interfacial width between low-density layer 3 and the loose monolayer of nanoparticles (layer 4). $\sigma_{4}$ is the interfacial width between the bulk of the electrolyte and the loose monolayer.

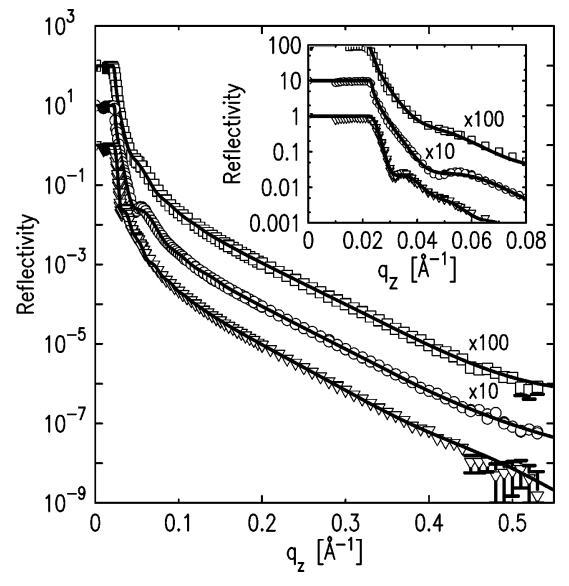

Figure 4. X-ray reflectivity as a function of the wave vector normal to the surface for the surface of the $\mathrm{NaOH}$-stabilized colloidal solutions: squares are for the solution of 5-nm particles; circles represent the solution of 7 -nm particles; and triangles represent the solution of 22-nm particles. Solid lines correspond to the four-layer model for the surface-normal structure. Inset: X-ray reflectivity at $q_{z}$ $<0.1 \AA^{-1}$.

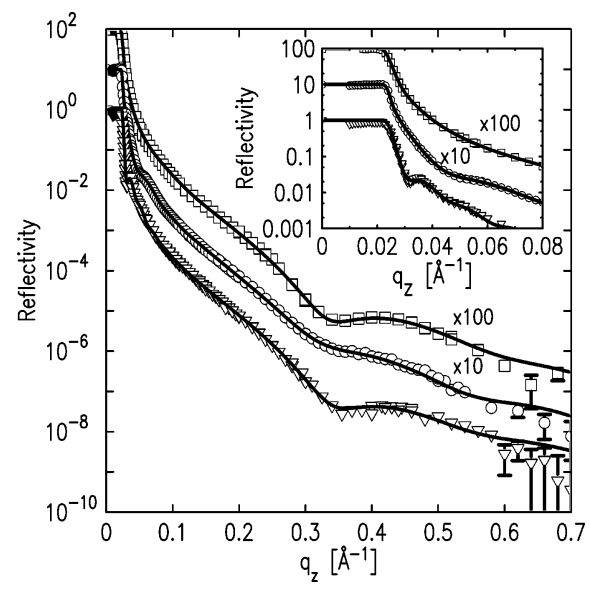

Figure 5. X-ray reflectivity as a function of the wave vector normal to the surface for the surface of the cesium-enriched colloidal solutions: squares represent the solution 5-nm particles; circles are for the solution 7-nm particles; and triangles are for the solution of 22-nm particles. Solid lines correspond to the four-layer model for the surfacenormal structure. Inset: the X-ray reflectivity at $q_{z}<0.1 \AA^{-1}$.

angle scattering, $c_{\mathrm{b}} \approx 10^{23} \mathrm{~m}^{-3}$ (Table 2). Alternatively, the small-angle scattering background can be measured from a bulk sample prepared in a narrow glass tube (see ref 26 for details).

Figures 4 and 5 show the X-ray reflectivity from the surfaces of sols stabilized by $\mathrm{NaOH}$ and the cesium-enriched solutions $\left(c_{\mathrm{Cs}}^{+} \geq c_{\mathrm{Na}}^{+}\right.$and $\left.\mathrm{pH}>11\right)$, respectively. The insets show data at $q_{z}<0.08 \AA^{-1}$ where reflectivity depends on the particles' size. At high angles, the sodium-stabilized and the cesium-enriched sols reflect light drastically differently: the reflectivity of the

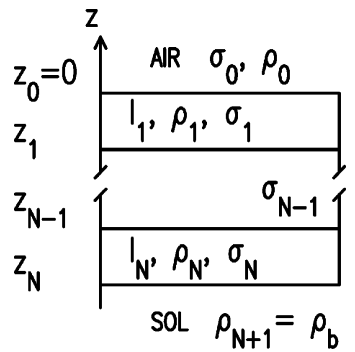

Figure 6. Parameters of the interfacial structure at the air/sol interface, chosen so that the interface coincides with the $x y$ plane $(z=0)$. Each layer has a thickness $l_{j}$ and an electron density $\rho_{j}$. In addition, four to five $\sigma_{j}$ parameters determine the interfacial width between slabs of electron density.

former monotonically decays at $q_{z}>0.1 \AA^{-1}$, whreas the latter's reflectivity oscillates in this interval. These data were obtained with the detector's vertical angular acceptance at $\Delta \beta=3.4 \times$ $10^{-2 \circ}$, and its horizontal acceptance at $\Delta \phi=0.8^{\circ}$.

\section{Models}

The X-ray reflectivity function, $R\left(q_{z}\right)$, in the first Born approximation can be represented as $R\left(q_{z}\right)=\left|F\left(q_{z}\right)\right|^{2} R_{F}\left(q_{z}\right)$, where $F\left(q_{z}\right)$ is the structure factor of the surface, and $R_{F}\left(q_{z}\right)$ is the Fresnel function, that is, the reflectivity from a sharp surface with no structure. ${ }^{38}$ I show below that the deviation of electron density in the transition region is such that the structure factor has a Hilbert phase and is defined by reflectivity only. ${ }^{39,40}$ Thus, the uncertainty in interpreting the data is related to the limited range of angles $\left(q_{z}^{\max } \approx 0.7 \AA^{-1}\right)$ covered in the experiment so that only interfacial models with spatial resolution $2 \pi / q_{z}^{\max } \approx$ $10 \AA$ can be tested.

To obtain the information about the surface-normal structure of the transition region, I used Parratt formalism that exactly solves X-ray reflectivity from a given structure. ${ }^{41}$ In the standard procedure, the interfacial structure is divided into $N$ layers (for example, ref 35), each having a thickness $l_{j}$, and electron density $\rho_{j}$ (see Figure 6). In addition, $\sigma_{j}$ parameters $(N+1)$ determine the interfacial width between the slabs of electron density. The structures are parametrized such that the air/sol interface coincides with the $x y$ plane $(z=0)$. I used the symmetrical error-function profiles of electron density across the interfaces so that the model's electron-density profile across the transition region is described by the following equation ${ }^{42,43}$

$$
\begin{array}{r}
\rho(z)=\frac{1}{2} \rho_{\mathrm{b}}+\frac{1}{2} \sum_{j=0}^{N}\left(\rho_{j+1}-\rho_{j}\right) \operatorname{erf}\left(\frac{t_{j}(z)}{\sigma_{j} \sqrt{2}}\right), \quad t_{j}(z)=z+\sum_{i=0}^{j} z_{i}, \\
\text { and } \operatorname{erf}(t)=\frac{2}{\sqrt{\pi}} \int_{0}^{t} \mathrm{e}^{-s^{2}} \mathrm{~d} s
\end{array}
$$


where $\rho_{N+1} \equiv \rho_{\mathrm{b}}$ is the electron density of the sol's bulk, $\rho_{0}=$ 0 and $z_{j}$, as in Figure 6.

The experimental findings for the surface of pure water revealed that the low limit for the parameter $\sigma_{0}$ was defined by the so-called capillary-wave roughness, $\sigma_{\text {cap }},{ }^{44,45}$ whose value is given by the detector's resolution, $q_{z}^{\max }=0.7 \AA^{-1}$ and a short wavelength cutoff in the spectrum of capillary waves

$$
\sigma_{\text {cap }}^{2}=\frac{k_{\mathrm{B}} T}{2 \pi \gamma} \ln \left(\frac{Q_{\mathrm{max}}}{Q_{\min }}\right)
$$

where $\gamma \approx 74 \mathrm{dyn} / \mathrm{cm}$ is the surface tension of sol surface measured by a Wilhelmy plate, and $Q_{\min }=q_{z}^{\max } \Delta \beta / 2$. Because the top part of the transition layer consists of water molecules and alkali ions, it is reasonable to use $Q_{\max }=2 \pi / a$ (where $a \approx$ $3 \AA$ is of the order of intermolecular distance). In these experiments, the calculated value for $\sigma_{\text {cap }}$ was as large as $2.6 \pm$ $0.2 \AA$, thereby setting the low limit for all $\sigma_{j}$ parameters. Any additional unspecified intrinsic structure of the interfaces can only increase $\sigma_{j}$.

The surface-normal structure of the sol stabilized by sodium hydroxide can be described satisfactorily by the three-layer model I proposed, which is discussed in detail for a hexane/sol interface in ref 27 . This model has up to 10 fitting parameters $(3 N+1)$. However, it predicts exceptionally high values for the interfacial widths of the compact layer (both $\sigma_{0}$ and $\sigma_{1}>$ $3.5 \AA$ ) that are markedly larger than the value given by eq 4 . Because, at high angles, the reflectivity from the liquid surface is $\sim \exp \left(-\sigma_{\text {cap }}^{2} q_{z}^{2}\right)$, the model probably cannot resolve some intrinsic structure of the compact layer. The contrast of the surface-normal structure of the cesium-enriched sols is such that it cannot be explained by this three-layer model: it does not describe satisfactorily the oscillations in the reflectivity at high angles (see Figure 5).

Nevertheless, reflectivity can be fitted by a 10-parameter 4-layer model wherein the compact layer is divided into 2 layers (layers 1 and 2) and $\sigma_{0}=\sigma_{1}=\sigma_{3}=\sigma_{\text {cap }}$ (see Figure 1). Here, the structure of the compact layer is described by the same number (four) of independent parameters as in the three-layer model but has better spatial resolution $(\sim 10 \AA)$. This model is appropriate for describing the ion finite-size effect in the compact layer. ${ }^{12,13}$ Models with more layers and/or number of fitting parameters insignificantly improve the quality of the fits. Therefore, more detailed information about the surface-normal structure can be obtained only by a large improvement in the spatial resolution of the experiment itself.

The solid lines in Figures 4 and 5 represent the four-layer model with the profiles shown in Figures 7 and 8; Table 2 lists its parameters. The error bars were estimated either from the uncertainties of the bulk properties or from the $\chi^{2}$ distribution versus the number of degrees of freedom, given by the number of data points.

\section{Electron Density Profile}

The compact layer can be considered, in the first approximation, as a mixture of water and alkali ions. For $\mathrm{Cs}^{+}$and $\mathrm{Na}^{+}$, the volume per one electron is as large as $v^{+} \approx 0.4 \AA^{3}{ }^{4}{ }^{46-48}$ Alternatively, the volume per one electron in a water molecule, $v_{\mathrm{w}} \approx 3 \AA^{3}$ and $>1 \AA^{3}$ in a hydroxyl ion. Therefore, the X-ray contrast of the compact layer is due to significantly higher density of electrons in alkali ions than in water molecules.

$\mathrm{X}$-ray reflectivity contains information about the surfacenormal structure of electron density, $\rho(z)$, averaged over a macroscopically large area $\left(\sim 0.5 \mathrm{~cm}^{2}\right)$. Because $v^{+} / v_{\mathrm{w}} \ll 1$, it

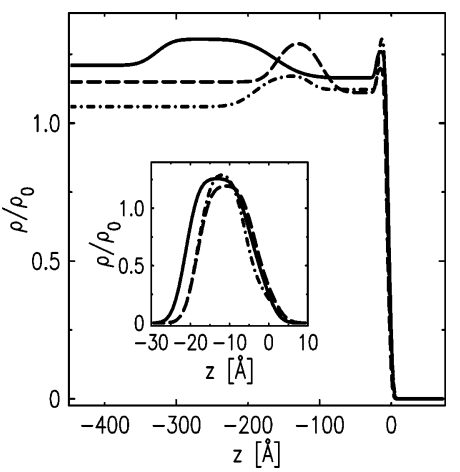

Figure 7. Normalized to the density of bulk water at normal conditions, $\rho_{0}=0.333 \mathrm{e}^{-} / \AA{ }^{3},{ }^{3}$ the electron-density profiles for the four-layer model for $\mathrm{NaOH}$-stabilized sols: solid line, solution of 22-nm particles; dashed line, solution of 7-nm particles; and dashed-dotted line, solution of 5-nm particles. The inset shows the distribution of electron density in the compact layer.

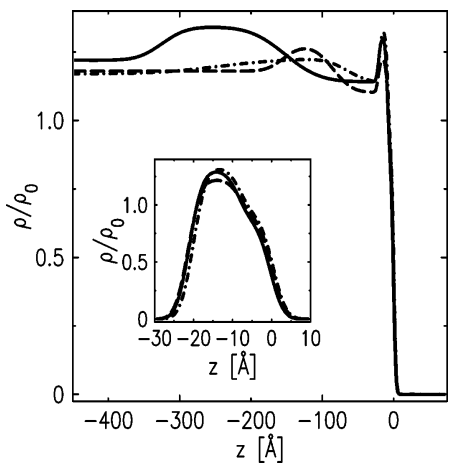

Figure 8. Normalized to the density of bulk water at normal conditions, $\rho_{0}=0.333 \mathrm{e}^{-} / \AA{ }^{3}{ }^{3}$ the electron-density profiles for the four-layer model for cesium-enriched sols: solid line, solution of 22-nm particles; dashed line, solution of 7-nm particles; and dashed-dotted line, solution of 5-nm particles. The inset shows the distribution of electron density in the compact layer.
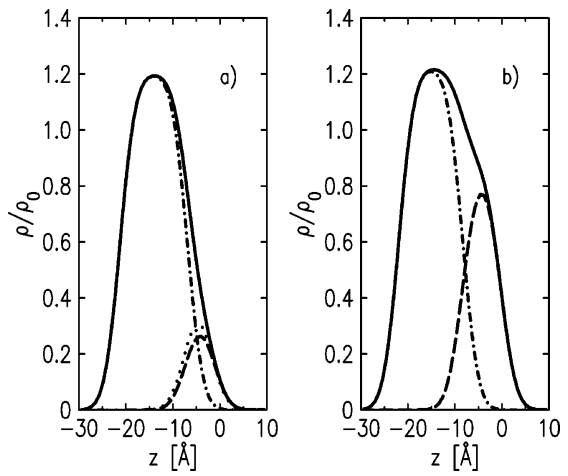

Figure 9. (a) $\mathrm{NaOH}-$ stabilized sols; (b) cesium-enriched sols. Solid lines are normalized to the density of bulk water $\left(\rho_{0}=0.333 \mathrm{e}^{-} / \AA^{3}\right)$ distributions of the electron density in the compact layer at the surface of the solution with 7-nm particles: The dashed lines and dashed-dotted lines are the distributions of the electron densities in layer 1 and layer 2 , respectively. The dotted line in Figure 8a shows the density of layer 1 for the sol with $c_{\mathrm{Na}}^{+} \approx 0.4 \mathrm{~mol} / \mathrm{L}(\mathrm{pH}>11)$.

is useful for further analysis to split into two terms the distribution of electron density $\rho_{\mathrm{c}}(z)$ along the $z$ axis per unit area in the compact layer

$$
\rho_{\mathrm{c}}(z)=\rho^{+}(z)+\rho_{\mathrm{w}}(z)
$$

where $\rho^{+}(z)$ is a distribution of electron density of alkali ions, and $\rho_{\mathrm{w}}(z)$ is a distribution of electron density of water molecules. 
TABLE 3: Integral Parameters of the Compact Layers ${ }^{a}$

\begin{tabular}{rccc}
\hline$D(\AA)$ & $\Gamma_{1}\left(\mathrm{~m}^{-2}\right) \times 10^{-20}$ & $\Gamma_{2}\left(\mathrm{~m}^{-2}\right) \times 10^{-20}$ & $\Phi_{2}\left(\mathrm{~m}^{-2}\right) \times 10^{-20}$ \\
\hline 50 & $2.5 \pm 0.3(0.7 \pm 0.4)$ & $5.3 \pm 0.5(5 \pm 2)$ & $1.0 \pm 0.2(1.0 \pm 0.3)$ \\
70 & $2.4 \pm 0.2(1.0 \pm 0.3)$ & $5.5 \pm 0.3(6 \pm 2)$ & $0.8 \pm 0.2(0.6 \pm 0.2)$ \\
220 & $2.3 \pm 0.2(1.0 \pm 0.4)$ & $5.5 \pm 0.5(7 \pm 1)$ & $0.8 \pm 0.2(0.6 \pm 0.2)$
\end{tabular}

${ }^{a} \Gamma_{1}$ and $\Gamma_{2}$ are the surface densities of electrons per unit area in layer 1 and 2, respectively; $\Phi_{2}$ is the total density of electrons of alkali ions per unit area in layer 1 . The numbers in parentheses correspond to the compact layers at the surface of $\mathrm{NaOH}$-stabilized sols.

$\rho_{\mathrm{c}}(z)$ can be calculated from eq 3 and Table 2 at $\rho_{3}=\rho_{4}=\rho_{\mathrm{b}}$ $=0$ so that the terms, which are not associated with the compact layer, are omitted (see the insets in Figures 7, 8, and 9). The constraint on the volume of the compact layer per unit area is the following

$$
v^{+} \rho^{+}(z) \mathrm{d} z+v_{\mathrm{w}} \rho_{\mathrm{w}}(z) \mathrm{d} z=f(z) \mathrm{d} z
$$

where $f(z)>0$ describes the averaged in- $x y$-plane distribution of the compact layer along the $z$ axis so that its volume per unit area or effective thickness is

$$
v_{0}=\int_{-\infty}^{+\infty} f(z) \mathrm{d} z
$$

Then, the total amount of electrons of alkali ions in the compact layer per unit area can be obtained from eqs 5 and 6 .

$$
\begin{aligned}
& \Phi=\int_{-\infty}^{+\infty} \rho^{+}(z) \mathrm{d} z \approx\left(\Gamma-\frac{v_{0}}{v_{\mathrm{w}}}\right)\left(1+\frac{v^{+}}{v_{\mathrm{w}}}\right) \\
& \text { with } \Gamma=\int_{-\infty}^{+\infty} \rho_{\mathrm{c}}(z) \mathrm{d} z
\end{aligned}
$$

In the next approximation, $v^{\mathrm{w}}$ can be treated as the volume per one electron of the solvent with the average electron-density $\sim \rho_{3}$. Then, $v^{\mathrm{w}}$ is $8-15 \%$ less than the volume per $\mathrm{H}_{2} \mathrm{O}$ molecule in water under normal conditions $v_{0}^{\mathrm{w}} \approx 3 \AA^{3}(\mathrm{~d} v / v=-\mathrm{d} \rho / \rho)$. The density of layer 3 ("surface water") can be slightly higher than the density of water at normal conditions, for example, due to electrostriction. ${ }^{26,49}$

When both $\delta v^{\mathrm{w}} / v_{0}^{\mathrm{w}}=\left(v^{\mathrm{w}}-v_{0}^{\mathrm{w}}\right) / v_{0}^{\mathrm{w}}$ and $v^{+} / v_{0}^{\mathrm{w}}$ are small, the following equation is obtained from eq 8 :

$$
\Phi \approx\left(\Gamma-\frac{v_{0}}{v_{0}^{\mathrm{w}}}\right)\left(1+\frac{v^{+}}{v_{0}^{\mathrm{w}}}\right)+\frac{v_{0}}{\left(v_{0}^{\mathrm{w}}\right)^{2}} \delta v^{\mathrm{w}}
$$

Equation 9 is the generalization of the equation that I derived earlier (ref 27).

Furthermore, eq 6 is not valid for layer 1 because its density is smaller than $\rho_{3}$ : the density of the water molecules in this layer must be much less than that in the bulk. Besides, the contribution of the layer $1\left(\sim \rho_{1}-\rho_{3}\right)$ to eq 9 is negative. Therefore, reasonably, eq 9 is applicable to layer 2 only, the effective thickness of which is $v_{0} \approx l_{2} \approx 13 \AA$ because $l_{2}>$ $2 \sigma_{\text {cap. }}$ Table 3 lists the integral parameters of the compact layer, where $\Gamma_{1}$ and $\Gamma_{2}$ are, respectively, the surface densities of electrons per unit area in layer 1 and 2; $\Phi_{2}$ is the total density of electrons of alkali ions per unit area in layer 2 . The numbers in parentheses correspond to the compact layer at the surface of sodium-hydroxide-stabilized sols.

I also compared these findings with those from a solution of 70 - $\AA$ particles with a high concentration of sodium hydroxide $c_{\mathrm{Na}}^{+} \approx 0.4 \mathrm{~mol} / \mathrm{L}(\mathrm{pH} \approx 11)$. The dotted line in Figure 8 a shows the density of layer 1 for this sol that is barely related to bulk concentration of sodium because it differs only slightly from the distribution of electron density in layer 1 of the solution with $c_{\mathrm{Na}}^{+} \approx 0.2 \mathrm{~mol} / \mathrm{L}$ (dashed line). In contrast, the integral $\Gamma_{1}$ for the cesium-enriched sols $\left(\sim l_{1} \rho_{1}\right)$ is three times larger than the $\Gamma_{1}$ for $\mathrm{NaOH}$-stabilized sols (compare the numbers in parentheses in Table 3). Hence, I assume that such a large difference is associated with the high concentration of $\mathrm{Cs}^{+}$in layer 1 with a surface density $\Gamma_{\mathrm{Cs}} \approx \Delta \Gamma_{1} /\left(Z_{\mathrm{Cs}}^{+}-Z_{\mathrm{Na}}^{+}\right) \approx 3 \times$ $10^{18} \mathrm{~m}^{-2}$, where $Z_{\mathrm{Cs}}^{+}=54$ and $Z_{\mathrm{Na}}^{+}=10$ are the numbers of electrons in $\mathrm{Cs}^{+}$and $\mathrm{Na}^{+}$, respectively.

Fortunately, the parameters of layer 2 in Table 2, and $\Phi_{2}$ in Table 3, are very similar for all sols I studied suggesting that layer 2 contains mostly $\mathrm{Na}^{+}$(see also Figure 9). Otherwise, $\mathrm{Cs}^{+}$ions would have to be present to build a layer with a significantly larger electron density than that listed in Table 2 to match the surface charge of the compact layer containing $\mathrm{Na}^{+}$. Thus, the surface density of $\mathrm{Na}^{+}$in layer 2 is $\Gamma_{\mathrm{Na}} \approx \Phi_{2} /$ $Z_{\mathrm{Na}}^{+} \approx 8 \times 10^{18} \mathrm{~m}^{-2}$, which is noticeably higher than the estimated surface density of sodium ions in the compact layer at the hexane-sol interface. ${ }^{27}$

\section{Discussion}

A. Surface Charge Density at the Sol's Surface. In the simplest model, the compact layer at the surface of the $\mathrm{NaOH}$ stabilized sol can be treated as a two-dimensional crystal of hydrated ions. The structure factor of such a two-dimensional lattice consists of a set of "Bragg rods" normal to the surface plane at $q_{z}=0$. The assumption that the grazing incidence diffraction's peak from the sol's surface arises due to spatial correlations between $\mathrm{Na}^{+}$at the length $\xi \approx 2 \pi / q_{x y}^{\prime} \approx 4 \AA$ demonstrates the good agreement between diffraction and reflectivity data for the surface density of the alkali ions, $\Gamma_{\mathrm{Na}}$ $\approx 1 / \xi^{2} \approx 6 \times 10^{18} \mathrm{~m}^{-2}$. However, the area per ion in the layer $\Gamma_{\mathrm{Na}}^{-1} \approx 20 \AA^{2}$ is much smaller than expected for a monolayer of hydrated $\mathrm{Na}^{+}\left[\left(2 a_{\mathrm{Na}}+2 d_{\mathrm{w}}\right)^{2} \approx 60 \AA^{2}\right.$ per ion]. In addition, the thickness of layer 2 is $50 \%$ larger than the diameter of the first hydration shell of the sodium ion $\left(2 a_{\mathrm{Na}}+2 d_{\mathrm{w}} \approx 8 \AA\right)$ : the charges in the compact layer should have a different spatial distribution from those in the monolayer of hydrated ions.

Because $c^{-} \ll c_{\mathrm{Cs}, \mathrm{Na}}^{+}$, the average charge per particle in the solutions, $Z_{0} \approx e N_{\mathrm{A}}\left(c_{\mathrm{Cs}}^{+}+c_{\mathrm{Na}}^{+}\right) / c_{\mathrm{b}}\left(N_{\mathrm{A}}\right.$ is the Avogadro constant) varies in accordance with the concentration of the base. The surface charge per unit area in the loose monolayer of nanoparticles is $e \Gamma^{-} \approx Z_{0} c_{4}^{-} l_{4} \approx 1 \mathrm{C} / \mathrm{m}^{2}$, wherein $c_{4}^{-}$is the concentration of particles $c_{4}^{-} \approx c_{\mathrm{b}}\left(\rho_{4}-\rho_{0}\right) /\left(\rho_{\mathrm{b}}-\rho_{0}\right) \approx 1.5 c_{\mathrm{b}}$ (see ref 9 for details). Alternatively, the surface charge per unit area in the compact layer is $e \Gamma^{+} \approx e\left(\Gamma_{\mathrm{Cs}}+\Gamma_{\mathrm{Na}}\right) \approx 2 \mathrm{C} / \mathrm{m}^{2}$ according to X-ray reflectivity data.

As discussed in ref 27, the measured width of the transition region at the sol's surface is comparable to the Debye screening length, $\Lambda_{\mathrm{D}} \approx 100-1000 \AA$, in the solution, which, under the Goy-Chapman theory, is a typical width of the diffuse layer. ${ }^{50}$ A reasonable assumption is that only ions at the distance $\sim \Lambda_{D}$ from the surface are involved in forming the transition region. Alternatively, building a negative countercharge of hydroxyl ions near a sol's surface with the surface density $e \Gamma^{+}-e \Gamma^{-} \approx$ $1 \mathrm{C} / \mathrm{m}^{2}$ would require the protonation of a very small fraction of the ionized silanol groups in the macroscopically wide region near the surface $\left[\left(\Gamma^{+}-\Gamma^{-}\right) / c^{-} \approx 1 \mu \mathrm{m}-10^{3} \mu \mathrm{m}\right.$ wide $\left.\gg \Lambda_{\mathrm{D}}\right] .{ }^{51}$ Therefore, the width of the transition region, $L \sim 20-40 \mathrm{~nm}$, established from $X$-ray reflectivity data, denotes the wide diffuse layer of nanoparticles $\left(\sim l_{2} c_{\mathrm{c}}^{+} / c^{+} \approx 30-80 \mathrm{~nm} \approx \Lambda_{\mathrm{D}}\right)$ rather than the transport of $\mathrm{OH}^{-}$ions from the bulk to the surface compact layer where the concentration of the hydroxyl ions can 
be as large as $\sim\left(\Gamma^{+}-\Gamma^{-}\right) / l_{2} \approx 10 \mathrm{~mol} / \mathrm{L}\left(\Gamma_{\mathrm{Na}}<\Phi_{2} / Z_{\mathrm{Na}}^{+}\right)$. Unfortunately, the contributions of $\mathrm{H}_{2} \mathrm{O}$ and $\mathrm{OH}^{-}$to the electron density of the compact layer cannot be reliably separated in the X-ray scattering data. Distinguishing precisely the ion-atom correlation functions at the sol's surface (for example, for a $\mathrm{Na}^{+}-\mathrm{O}$ pair) from the intensity of X-ray grazing incidence diffraction could afford further useful information on the mixing of hydroxyl and sodium ions in the compact layer. A quantitative model of the transition region that would account for chemical equilibrium between the hydroxyl ions and silanol groups would also be useful in elucidating the nature of the negative countercharge in the transition region.

B. Surface Tension. At equilibrium, the energy gain, $w^{-}$, for disassociating the silanol group at the silica's surface, for example, at $\mathrm{pH}=11$, is $w^{-} \approx-k_{\mathrm{B}} T \ln \left(c^{+} / c^{-}\right) \approx-6 k_{\mathrm{B}} T$ per ion. ${ }^{51}$ This defines roughly the "binding energy", $w_{\mathrm{b}}^{+}$, of the alkaline ion in the sol's bulk: $w_{\mathrm{b}}^{+} \approx w^{-}$. The adsorption of alkali ions in the surface compact layer is associated with the energy gain, $w^{+}$, which is comparable to $w^{-}: w^{+} \approx-k_{\mathrm{B}} T \ln ($ $\left.c_{\mathrm{c}}^{+} / c^{+}\right) \approx-4 k_{\mathrm{B}} T$ per ion, where the volume concentration of alkali ions in the compact layer, $c_{\mathrm{c}}^{+}$, ranges from $c_{\mathrm{c}}^{+} \approx \Gamma^{+} / l_{2} \approx$ $17 \mathrm{~mol} / \mathrm{L}$ to $\left.c_{\mathrm{c}}^{+} \approx 1 / \xi^{3} \approx 26 \mathrm{~mol} / \mathrm{L}\right)$. Therefore, the energy cost, $\Delta w$, to bring a hydrated alkaline ion from the sol's bulk to the air/sol interface is $\Delta w=w^{+}-w_{b}+\approx-k_{\mathrm{B}} T \ln ($ $\left.c_{\mathrm{c}}^{+} c^{-} /\left(c^{+}\right)^{2}\right) \approx 2 k_{\mathrm{B}} T$. This value is comparable to $\Delta w$ for hydrated $\mathrm{Na}^{+}$at the air/water interface (see Figure 2 in ref 14). If the hydrosol's surface consists of hydrated alkaline ions, then the total energy toll, $\gamma$, to create $1 \mathrm{~m}^{2}$ of the sol's surface is $\gamma$ $\approx \Gamma^{+} \Delta w^{+} \approx 90 \mathrm{~mJ} / \mathrm{m}^{2}$. The experimental value for the surface tension $\gamma=74 \mathrm{~mJ} / \mathrm{m}^{2}$ agrees exceptionally well with this rough estimation. Finally, the small surface density of $\mathrm{Na}^{+}, \Gamma^{+} \approx 2$ $\times 10^{18}-6 \times 10^{18} \mathrm{~m}^{-2}$, at the $n$-hexane/silica sol interface (up to 4 times less than at the air/sol interface) can be explained by the higher value of $\Delta w \approx 3 k_{\mathrm{B}} T\left(\gamma \approx 40 \mathrm{~mJ} / \mathrm{m}^{2}\right){ }^{27}$

C. Selective Adsorption of Alkali Ions in Layer 1 and Layer 2. The adsorption of alkaline ions at the surface of the sol lowers the electrostatic energy of the transition region: the interaction of the compact layer is attractive to either the nanoparticles or the "image charge" they induce. According to Kharkats and Ulstrup, ${ }^{12,13}$ the electrostatic Gibbs energy, $G(h)$, of an alkali ion sited at the planar boundary between two dielectric media is

$$
G(h=0)=\frac{1}{4 \pi \epsilon_{0}} \frac{e^{2}}{\left(\epsilon_{1}+\epsilon_{2}\right)} \frac{1}{a}
$$

where $a$ is the ion's radius. The radius of $\mathrm{Cs}^{+}, a_{\mathrm{Cs}} \approx 1.7 \AA$, is considerably larger than the radius of $\mathrm{Na}^{+}, a_{\mathrm{Na}} \approx 1 \AA$. Therefore, in accordance with eq 10, the direct adsorption of the former at the sol's surface is more favorable than the adsorption of the latter: hence, cesium ions will replace sodium ions at the surface because this is associated with a negative change in Gibbs energy $(\Delta G<0)$.

When $h \geq a$, the finite size effect in $G(h)$ is described the following term: ${ }^{14,21}$

$$
\frac{e^{2}}{32 \pi \epsilon_{0} \epsilon_{1} a}\left(\frac{\epsilon_{1}-\epsilon_{2}}{\epsilon_{2}+\epsilon_{2}}\right)^{2}\left[\frac{2}{1-(2 h / a)^{2}}+\frac{a}{2 h} \ln \frac{2 h+a}{2 h-a}\right]
$$

At $h \gg a$, it is reduced to

$$
\approx \frac{e^{2}}{16 \pi \epsilon_{0} \epsilon_{1}} \frac{a^{3}}{h^{4}}
$$

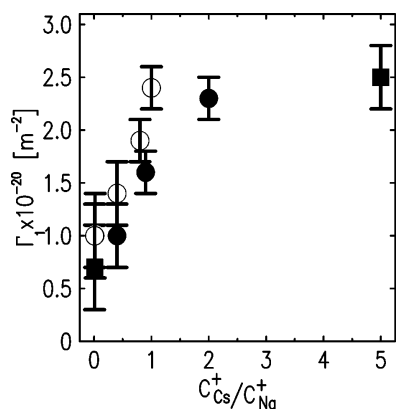

Figure 10. Dependence of $\Gamma_{1}$ on $c_{\mathrm{Cs}}^{+} / c_{\mathrm{Na}}^{+}$in the cesium-enriched sols: squares are for the solution of 5-nm particles; circles are for the solution of 7-nm particles; and dots are for the solution of 22-nm particles.

because $\epsilon_{1} \gg \epsilon_{2}$. This explains the constant density of layer 2 ( $h \approx 10 \AA$ ) for all the sols I studied: that is, the accumulation of small $\mathrm{Na}^{+}$ions at $h \gg 1 \AA$ is more favorable than that for large $\mathrm{Cs}^{+}$ions $(\Delta G<0)$. Therefore, ionic size drives the selective adsorption of alkali ions at layer 1 and layer 2 .

My analysis shows that the difference between the reflectivity at high angles of $\mathrm{NaOH}$-stabilized sols and cesium-enriched sols depends mostly on the density of layer 1 . Its thickness, $l_{1}$, is approximately equal to the diameter of the first hydration shell of $\mathrm{Cs}^{+}, 2 a_{\mathrm{Cs}}+2 d_{\mathrm{w}}$, where $d_{\mathrm{w}} \approx 3 \AA$ is a water molecule's diameter. The estimated density of the directly adsorbed ions $\Gamma_{\mathrm{Cs}}$ in layer 1 corresponds to a monolayer with an area per $\mathrm{Cs}^{+}$ $\approx 33 \AA .^{2}$

My X-ray reflectivity data for the cesium-enriched sols fully agrees with the earlier results of many authors: less hydrated cesium ions adsorb more strongly at the silica surface than do strongly hydrated sodium ions. ${ }^{31,55-59}$ When $c_{\mathrm{Cs}}^{+}<c_{\mathrm{Na}}^{+}(\mathrm{pH}<$ $11)$, the reflectivity at high angles depends upon $c_{\mathrm{Cs}}^{+} / c_{\mathrm{Na}}^{+}$: then, cesium ions preferentially adsorb to the silica surface (in the bulk) rather than at the sol's surface. However, when $c_{\mathrm{Cs}}^{+} \geq c_{\mathrm{Na}}^{+}$ $(\mathrm{pH}>11)$ the density of layer $\Gamma_{1}$ saturates and adsorption becomes independent of $c_{\mathrm{Cs}}^{+} / c_{\mathrm{Na}}^{+}$(see Figure 10).

\section{Conclusions}

(1) According to my X-ray reflectivity experiment, the surface-normal structure of the compact layer at the surface of the silica hydrosol is almost independent of the size of the particles and concentration of $\mathrm{NaOH}$ in the suspension. The estimated surface density of sodium ions adsorbed at the compact layer is up to 4 times larger than it is at the $n$-hexane/ sol interface, which I studied in previous experiments. ${ }^{27}$ The position of the grazing incidence diffraction peak at $q_{x y}^{\prime} \approx 1.5$ $\AA^{-1}$, observed at the air/hydrosol interface, corresponds to the spatial correlations between hydrated $\mathrm{Na}^{+}$in the compact layer at the length $\xi \approx 4 \AA$. The large difference between the number of adsorbed ions at the air/sol and the hexane/sol interfaces can be related to the difference between the energy costs to bring a single hydrated $\mathrm{Na}^{+}$ion to these interfaces.

(2) I also studied hydrosols stabilized by a mixture of $\mathrm{NaOH}$ and $\mathrm{CsOH}$, with the total bulk concentration of alkali ions ranging from 0.1 to $0.7 \mathrm{~mol} / \mathrm{L}$. I demonstrated that $\mathrm{Na}^{+}$and $\mathrm{Cs}^{+}$, which have different ion radii, interact differently with the sol's surface. The structure of the compact layer can be described by a two-layer model, that is, an $\sim 8$ - $\AA$-thick layer of directly adsorbed hydrated alkali ions with a surface concentration of $\sim 3 \times 10^{18} \mathrm{~m}^{-2}$, and $\mathrm{a} \sim 13$ - $\AA$-thick layer with a surface concentration of sodium ions of $\sim 8 \times 10^{18} \mathrm{~m}^{-2}$. In cesiumenriched sols, $\mathrm{Cs}^{+}$ions preferentially adsorb in the first layer, replacing $\mathrm{Na}^{+}$; their density in the second layer does not depend 
on the presence of cesium in the sol. The difference in the adsorption of $\mathrm{Cs}^{+}$and $\mathrm{Na}^{+}$ions can be explained by the ionsize-dependent term in the electrostatic Gibbs energy equation derived earlier by Kharkats and Ulstrup. ${ }^{12,13}$

Acknowledgment. Use of the National Synchrotron Light Source, Brookhaven National Laboratory, was supported by the U.S. Department of Energy, Office of Science, Office of Basic Energy Sciences, under Contract No. DE-AC02-98CH10886. $\mathrm{X} 19 \mathrm{C}$ is partially supported through funding from the ChemMatCARS National Synchrotron Resource, the University of Chicago, and the University of Illinois at Chicago. I thank Grace Davison for providing solutions of colloidal silica. I thank Professor Mark L. Schlossman and Professor Vladimir I. Marchenko for valuable discussions. I also thank Avril Woodhead and Anna I. Lygina.

\section{References and Notes} 1982.

(1) Franks, F.; Mathias, S. E. Biophysics of Water; Wiley: Chichester,

(2) Demlov E. V; Demlov S. S. Phase Transfer Catalysis, 2nd ed.; Verlag Chemie: Weinheim, 1983.

(3) Starks, C. M.; Liotta, C. L.; Halpern, M. Phase Transfer Catalysis; Chapman \& Hall: New York, 1994.

(4) Adamson, A. W. Physical Chemistry of Surfaces, 3rd ed.; John Wiley \& Sons: New York, 1976.

(5) Everett, D. H. Basic Principles of Colloid Science; The Royal Society of Chemistry: London, 1988.

(6) Finlayson-Pitts, B. J. Chem. Rev. 2003, 103, 4801.

(7) Rieger, Ph. H. Electrochemistry, 2nd ed,; Chapman \& Hall: New York, 1994.

(8) Frumkin, A. N. Z. Phys. Chem. 1924, 109, 34.

(9) Frumkin, A. N. Sbornik rabot po chistoy i prikladnoy khimii; Scientific Chemical Technical Publishing House: Petrograd, 1924; pp 106126 (in Russian).

(10) Wagner, C. Phys. Z. 1924, 25, 474.

(11) Onsager, L.; Samaras, N. N. T. J. Chem. Phys. 1934, $2,528$.

(12) Kharkats, Y. I.; Ulstrup, J. J. Electroanal. Chem. 1991, 308, 17.

(13) Ulstrup, J.; Kharkats, Yu. I. Russ. J. Electrochem. 1993, 29, 299.

(14) Markin, V. S.; Volkov, A. G. J. Phys. Chem. B 2002, 106, 11810.

(15) Toney, M. F.; Howard, J. N.; Richer, J.; Borges, G. L.; Gordon, J. G.; Melroy, O. R.; Wiesler, D. G.; Yee, D.; Sorensen, L. B. Nature (London) 1994, 368, 444.

(16) Wang, J.; Ocko, B. M.; Davenport, A. J.; Isaacs, H. S. Phys. Rev. B 1992, 46, 10321.

(17) Andrews, S. R.; Cowley, R. A. J. Phys. C: Solid State Phys. 1985, $18,6427$.

(18) Robinson, I. K. Phys. Rev. B 1986, 33, 3830.

(19) Feidenhansl, R. Surf. Sci. Rep. 1989, 10, 105.

(20) Kaganer, V. M.; Möhwald, H.; Dutta, P. Rev. Mod. Phys. 1999, 71,779 .

(21) Benjamin, I. Chem. Rev. 1996, 96, 1449.

(22) Jungwirth, P.; Tobias D. J. Chem. Rev. 2006, 106, 1259.

(23) Chang, T. M.; Dang, L. X. Chem. Rev. 2006, 106, 1305.

(24) Luo, G.; Malkova, S.; Yoon, J.; Schultz, D. G.; Lin, B.; Meron,

M.; Benjamin, I.; Vanysek, P.; Schlossman, M. L. Science 2006, 311, 216.

(25) (a) Graham, T. Trans. R. Soc. (London) 1861, 151, 183. (b) Ryznar, J. W. In Colloidal Chemistry: Theoretical and Applied; Alexander, J. B., Ed.; Reinhold Publishing Corporation: New York, 1946; Vol. VI.

(26) Tikhonov, A. M. J. Phys. Chem. B. 2006, 110, 2746.

(27) Tikhonov, A. M. J. Chem. Phys. 2006, 124, 164704.

(28) Landau, L. D.; Lifshitz, E. M. Electrostatics of Dielectrics. Electrodynamics of Continuous Media, 2nd. ed.; Pergamon: New York, 1984; Chapter II.

(29) Madsen, A.; Konovalov, O.; Robert, A.; Grubel, G. Phys. Rev. E.

2001, 64, 61406.

(30) Vorotyntsev, M. A.; Ermakov, Y. A.; Markin, V. S.; Rubashkin, A. A. Russ. J. Electrochem. 1993, 29, 730.
(31) Depasse, J.; Watillon, A. J. Colloid Interface Sci. 1970, 33, 430.

(32) The diameters of silica particles are in accordance with Grace Davison's data.

(33) Schlossman, M. L.; Synal, D.; Guan, Y.; Meron, M.; SheaMcCarthy, G.; Huang, Z.; Acero, A.; Williams, S. M.; Rice, S. A.; Viccaro, P. J. Rev. Sci. Instrum. 1997, 68, 4372.

(34) Usually, it takes about two hours at beamline X19C to measure the reflectivity curve from the sol's surface $\left(R\left(q_{z}^{\max }\right) \approx 10^{-9}\right.$ at $q_{z}^{\max } \approx 1$ $\left.\AA^{-1}\right)$.

(35) Tolan, M. X-ray Scattering from Soft-Matter Thin Films; Springer Tracts in Modern Physics, 148; Springer: New York, 1999.

(36) Hura, G.; Sorenson, J. M.; Glaeser, R. M.; Head-Gordon, T. J. Chem. Phys. 2000, 113, 9140.

(37) Guinier, A. X-ray Diffraction in Crystals, Imperfect Crystals and Amorphous Bodies; Dover Publications, Inc: New York, 1994.

(38) Als-Nielsen, J. Topics in Current Physics, Structure and Dynamics of Surfaces; Springer-Verlag: Berlin, 1986; Vol. 2.

(39) Clinton, W. L. Phys. Rev. B 1993, 48, 1.

(40) The profile yields a reflectivity from which the phase can be reconstructed unambiguously if $\left|\Delta \rho_{n}\right|>\Sigma_{m \neq n}\left|\Delta \rho_{m}\right|$, where the density contrasts $\Delta \rho_{m}=\rho_{m}-\rho_{m-1}$ between layers of the interfacial model (see Figure 6). ${ }^{39}$ This condition is fulfilled for the four-layer model discussed in this paper.

(41) Parratt, L. G. Phys. Rev. 1954, 95, 359.

(42) Buff, F. P.; Lovett, R. A.; Stillinger, F. H. Phys. Rev. Lett. 1965, 15,621 .

(43) Tidswell, I. M.; Ocko, B. M.; Pershan, P. S.; Wasserman, S. R.; Whitesides, G. M.; Axe, J. D. Phys. Rev. B 1990, 41, 1111.

(44) Braslau, A.; Pershan, P. S.; Swislow, G.; Ocko, B. M.; Als-Neilsen, J. Phys. Rev. A 1988, 38, 2457.

(45) Schwartz, D. K.; Schlossman, M. L.; Kawamoto, E. H.; Kellogg, G. J.; Pershan, P. S.; Ocko, B. M. Phys. Rev. A 1990, 41, 5687.

(46) The volume of the $\mathrm{Cs}^{+}$ion (contains 54 electrons) is as large as $\sim 21 \AA^{3}$ so that $v^{+} \approx 0.4 \AA^{3}$. For $\mathrm{Na}^{+} v^{+} \approx 0.4 \AA^{3}$ because the sodium ion contains only 10 electrons and its volume is $\sim 4 \AA^{3}$. The volume of $\mathrm{OH}^{-}$ (contains 10 electrons) is $\sim 11 \AA^{3}$. The values for ionic radii can be found, for example, in refs 47 and 48 .

(47) Huheey, J. E.; Keiter, E. A.; Keiter, R. L. Inorganic Chemistry: Principles of Structure and Reactivity, 4th ed.; Harper Collins: New York, 1993.

(48) Williams, A. F. A Theoretical Approach to Inorganic Chemistry; Springer-Verlag: Berlin, 1979

(49) Danielewicz-Ferchmin, I.; Ferchmin, A. R. J. Phys. Chem. 1996, 100,17281

(50) Because most of the akali ions in the sol's bulk must concentrate near the silica surface, the Debye screening length in the solution between particles (at $\mathrm{pH}=9-11)$ can be as large as $\Lambda_{\mathrm{D}}=\sqrt{\epsilon_{0} \epsilon_{1} k_{\mathrm{B}} T /\left(c^{-} N_{\mathrm{A}} e^{2}\right)} \approx$ $100-1000 \AA$, where $N_{\mathrm{A}}$ is the Avogadro constant.

(51) The chemical equilibrium in the hydrosol at $\mathrm{pH} \geq 9$ is due to the protonation-deprotonation of the silanol groups at the silica surface. ${ }^{52-54}$ At pHs between 9 and 12, the bulk concentration of $\mathrm{OH}^{-}$is smaller by 2 to 4 orders of magnitude than the bulk concentration of alkaline ions. At equilibrium, the desorption of the hydroxyl ion (the protonation of the ionized silanol group) at the silica's surface (in the bulk) is associated with the energy barrier $\sim k_{\mathrm{B}} T \ln \left(c^{+} / c^{-}\right)$. The adsorption of the free hydroxyl ions with the surface charge density of $\sim 1 \mathrm{C} / \mathrm{m}^{2}$ at the sol's surface would require the absolute depletion of the macroscopically wide region near the surface (1-100 $\mu \mathrm{m}$ wide $\gg \Lambda_{\mathrm{D}}$ ) with a subsequent inevitable change in the bulk chemical equilibrium. A small fraction of the silanol groups in this region would have to be protonated in order to restore the equilibrium.

(52) Parks, G. A. Chem. Rev. 1965, 65, 177.

(53) Yoon, R. H.; Salman, T.; Donnay, G. J. Colloid Interface Sci. 1979 $70,483$.

(54) Dumont, F.; Warlus, J.; Watillon, A. J. Colloid Interface Sci. 1990, 138, 543.

(55) Allen, L. H.; Matijevic, E. J. Colloid Interface Sci. 1969, 31, 287.

(56) Tadros, T. F.; Lyklema, J. J. Electroanal. Chem. 1968, 17, 267.

(57) Milonjic, S. K. Colloids Surf. 1992, 63, 113.

(58) Sonnefeld, J.; Gobel, A.; Vogelsberger W. Colloid Polym. Sci. 1995, 273, 926.

(59) Colic, M.; Fisher, M. L.; Franks, G. V. Langmuir 1998, 14, 6107. 